\title{
Transposition of Tibialis Anterior to the Cuboid after Tarsometatarsal Amputation
}

\author{
${ }^{1}$ Pradeep Moonot, ${ }^{2}$ Arun Bal, ${ }^{3}$ Ammar Qureshi
}

\begin{abstract}
Forefoot and midfoot amputees are prone to develop nonhealing ulcers at the closure site. This mainly occurs due to the limited dorsiflexion ability of the ankle and associated loss of protective function as seen in diabetics. One of the proposed methods to prevent these postamputation ulcers includes tendoachilles lengthening during the procedure. We describe three cases with nonhealing ulcers on the lateral aspect after a midfoot amputation. All of the cases underwent a tibialis anterior transposition to the lateral aspect of the foot to correct the deformity and at the same time also achieved healing of the ulcers.
\end{abstract}

Keywords: Amputation, Diabetic foot, Midfoot, Tendon transfer, Tibialis anterior, Transmetatarsal.

How to cite this article: Moonot P, Bal A, Qureshi A. Transposition of Tibialis Anterior to the Cuboid after Tarsometatarsal Amputation. J Foot Ankle Surg (Asia-Pacific) 2016;3(2): 107-110.

\section{Source of support: Nil \\ Conflict of interest: None}

\section{INTRODUCTION}

A diabetic patient has a 12 to $25 \%$ lifetime risk of developing a foot ulcer, ${ }^{1}$ making it the most common cause of diabetic patients' hospitalization. ${ }^{2}$ Following amputation, these patients are at risk of recurrent ulcers. Apart from decreased sensations in the foot, decreased dorsiflexion has been implicated in the cause of recurrent ulcers. The limited dorsiflexion prevents the leg from rolling over the foot during the late stance phase of gait, leading to raised plantar pressures. ${ }^{3-6}$

Tendoachilles lengthening is done for recurrent ulcers so as to increase the range of dorsiflexion that will reduce the plantar pressures, hence reducing the skin breakdown. ${ }^{4-7}$ In the current series, tendoachilles lengthening was not done for any of the cases. All the stumps developed nonhealing ulcers. The feet also went into supination owing to the tendon imbalance.

Based on studies by Turan, ${ }^{8}$ the tibialis anterior tendon was transposed to the lateral cuneiform. This transposition

\footnotetext{
${ }^{1,2}$ Consultant, ${ }^{3}$ Senior Registrar

${ }^{1,3}$ Department of Orthopaedics, S. L. Raheja Fortis Hospital Mumbai, Maharashtra, India

${ }^{2}$ Department of Diabetic and Foot Surgery, S. L. Rahega Hospital, Mumbai, Maharashtra, India
}

Corresponding Author: Pradeep Moonot, Consultant Department of Orthopaedics, S. L. Raheja Fortis Hospital Mumbai, Maharashtra, India, e-mail: drmoonot@gmail.com not only addressed the problem of the supination deformity but also helped in promoting the ulcer healing.

\section{MATERIALS AND METHODS}

We studied three patients diagnosed with diabetic foot where all of them had undergone a transmetatarsal amputation. Postoperatively, at an average of 1 year after the procedure, these patients presented with a nonhealing ulcer at the site of wound closure. Local examination revealed a supple foot with good range of dorsiflexion at the ankle and a correctable supination deformity of the foot.

\section{Case 1 (Figs $1 \mathrm{~A}$ to $\mathrm{F}$ )}

\section{Surgical Methods}

The patients were kept supine with a bump under the ipsilateral buttock. Positioning must allow exposure to medial and lateral foot and anterior aspect of distal leg. A dorsomedial incision is made over the insertion of the tibialis anterior into the navicular (Fig. 2). Pneumatic tourniquet was used in all the three cases. The insertion of tibialis anterior was sharply released from the navicular up to the periosteum to get the maximum length. A second incision was placed in the distal aspect of the anterior leg just proximal to the extensor retinaculum (Fig. 3).

The tibialis anterior tendon was brought out from the second incision. A whipstitch was used to tag the end of the tendon using No 5 polyester sutures (Fig. 4). A third incision was made dorsolaterally over the cuboid bone. The tendon was retrieved and delivered through this third incision. A guidewire was passed from the dorsolateral to plantar-medial surface of the cuboid (Figs 5 and 6 ); after confirming the guidewire position under fluoroscopy, the canal was reamed with a $6 \mathrm{~mm}$ cannulated drill bit. The prepared tendon was passed through the canal out to the plantar aspect of the foot. Then the interference screw was advanced from the dorsal to plantar surface to attach the tendon to the bone. While tightening the interference screw, maximum traction was given to the tendon and the foot was kept in dorsiflexion and eversion. The tendon was sutured to the surrounding tissue with absorbable sutures.

After irrigation, the surgical wounds were closed in layers (Fig. 7). The ankle was immobilized in a belowknee slab in eversion and dorsiflexion which after staple removal was converted to a below-knee cast for 4 weeks. 

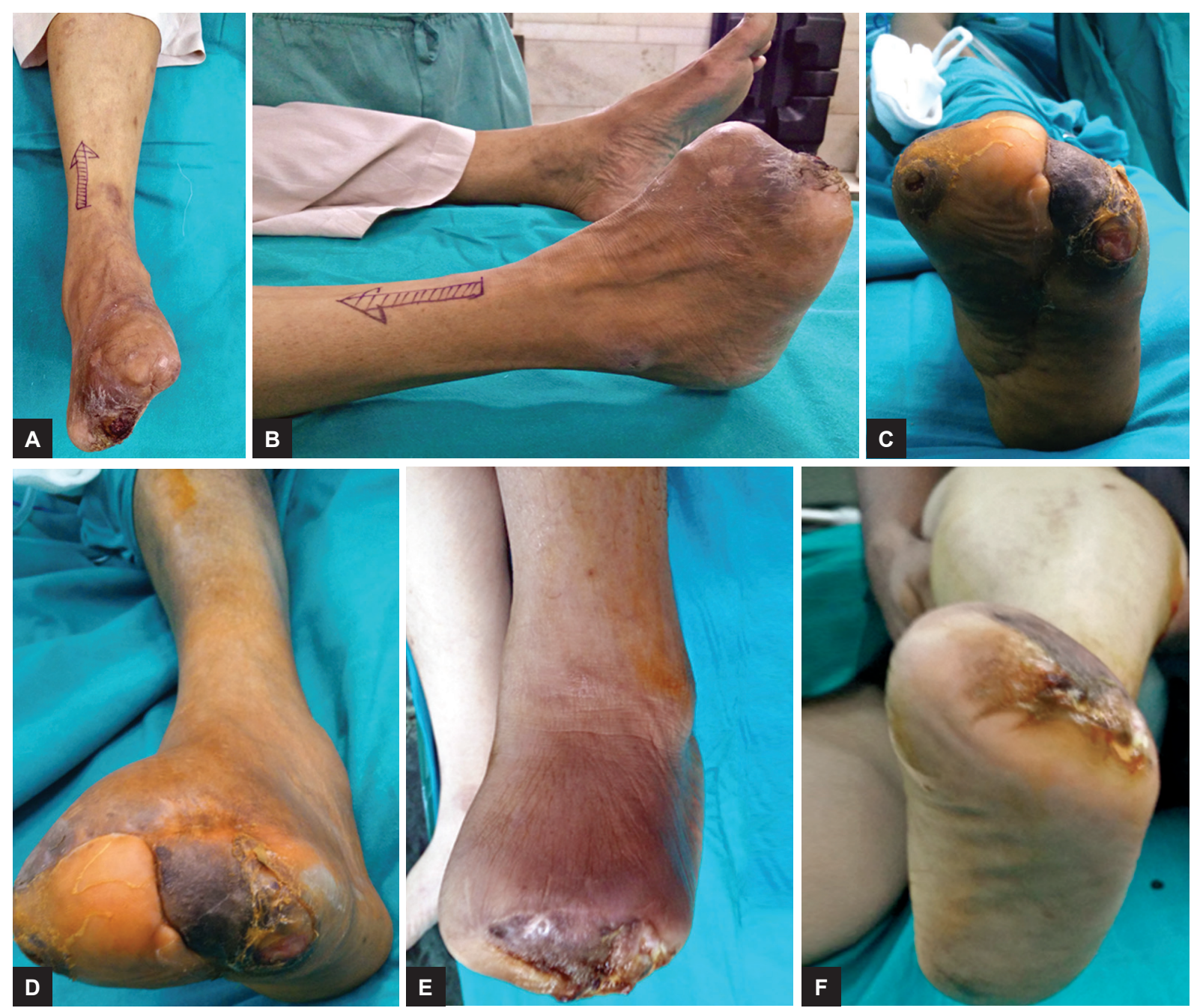

Figs $1 \mathrm{~A}$ to $\mathrm{F}:$ Nonhealing ulcer on the lateral and plantar aspect of the stump

After this period, ankle mobilization was done. The patient was kept strictly nonweight bearing for 6 weeks. When ankle mobilization was started, gradual weight bearing with restricted plantar flexion was allowed for 2 to 3 weeks. Follow-up was done at 2, 6 weeks, 3, and 6 months.

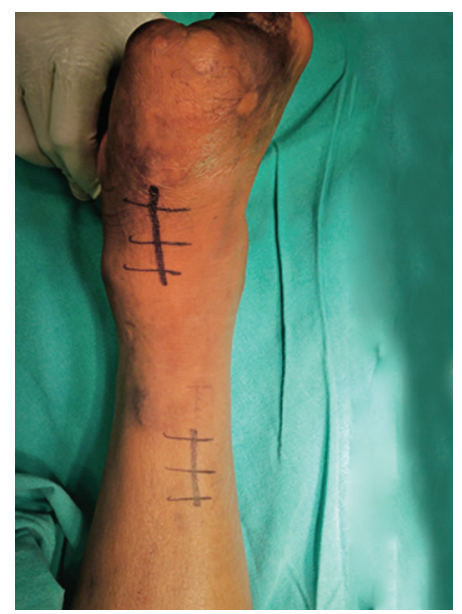

Fig. 2: Incision marked for the tibialis anterior tendon transfer in the supine position

\section{RESULTS}

Postoperatively, good range of motion was retained in the ankle joint. Regular dressings were done for the ulcers in the three patients. The ulcers had completely healed in an average of 2 to 3 months following the procedure and regular dressings (Figs 8 and 9).

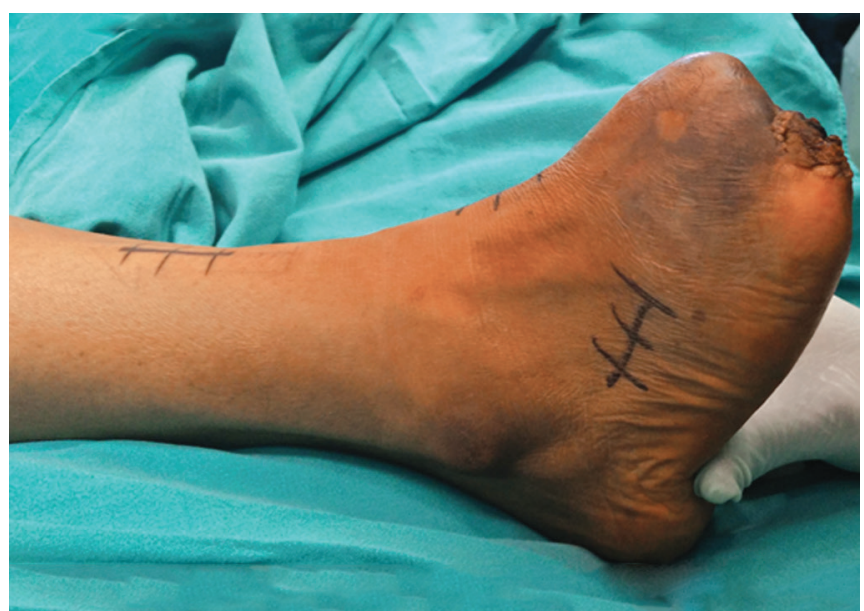

Fig. 3: Incision marked for the tibialis anterior tendon transfer from the lateral aspect 


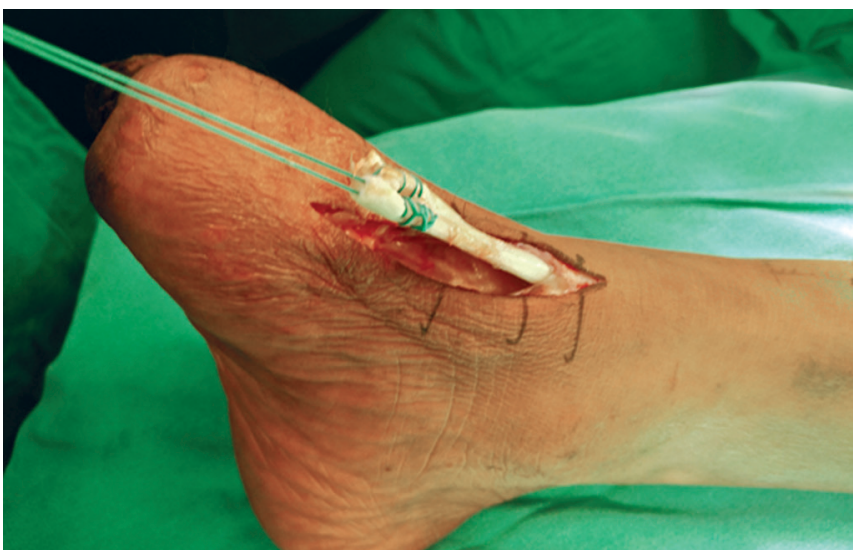

Fig. 4: Tibialis tendon harvested and stitched with non absorbable suture

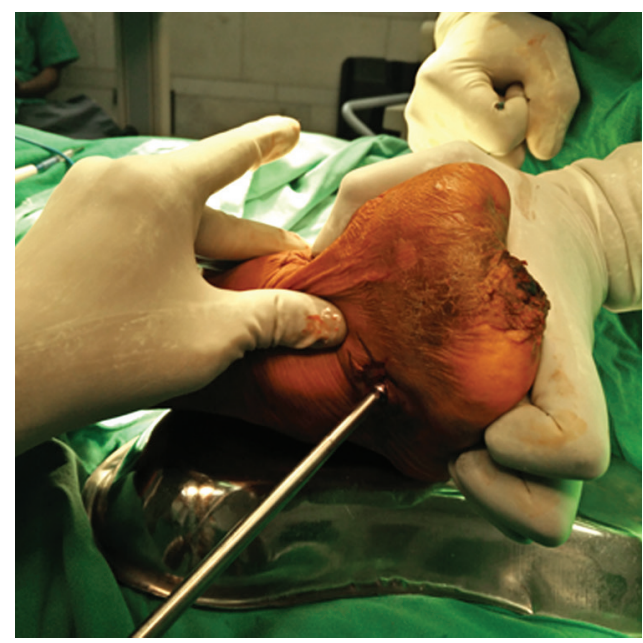

Fig. 6: Tendon fixed with a biointerferance screw

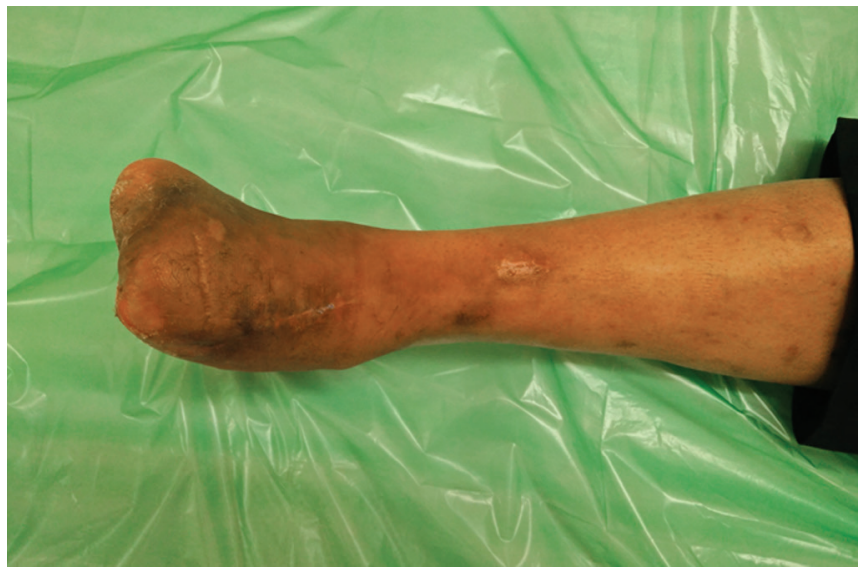

Fig. 8: Three months after surgery the wound has healed completely-dorsal view

\section{DISCUSSION}

There are many options for treating the nonhealing ulcers. These include refashioning the stump, split transfer of the tibialis anterior, gastrocsoleus recession, and transposition of tibialis anterior to the lateral cuneiform or cuboid. Tendoachilles lengthening has been shown to reduce the risk of ulceration and improve healing of diabetic foot ulcers. ${ }^{4-7}$

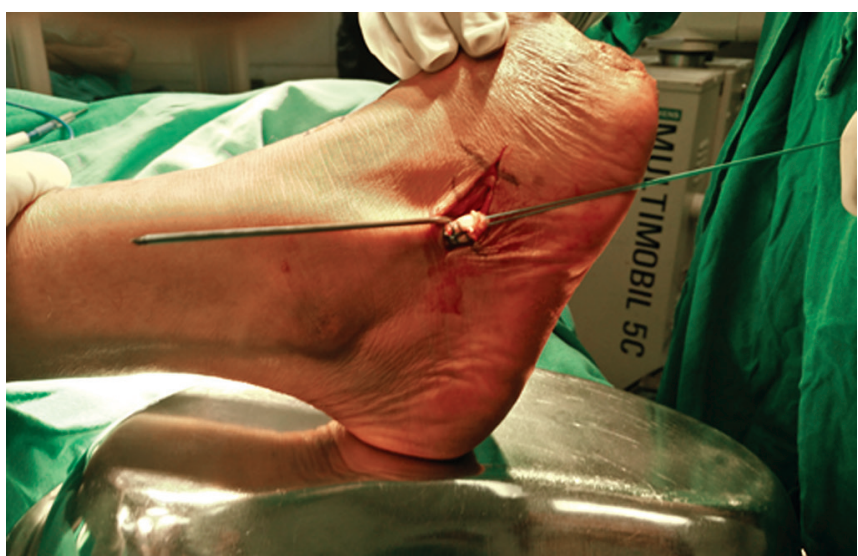

Fig. 5: Tendon routed through the leg and taken out in the lateral wound on the midfoot

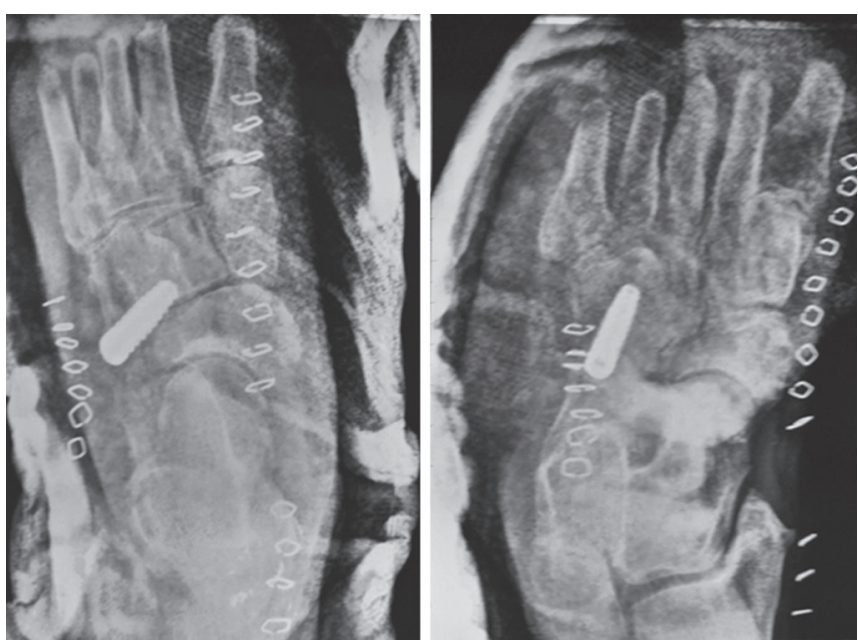

Fig. 7: Postoperative X-rays

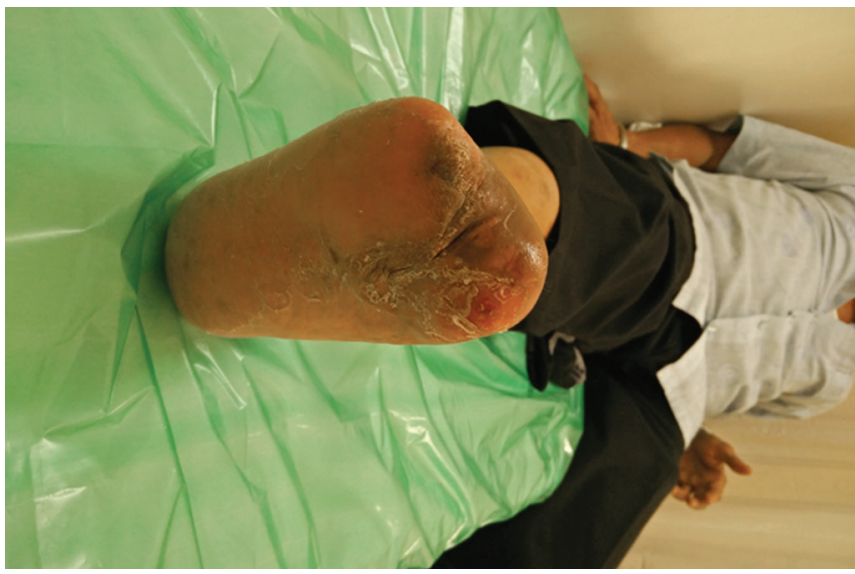

Fig. 9: Three months after surgery the wound has healed completely_plantar view

The procedure increases the range of dorsiflexion and at the same time temporarily reduces the plantar flexor peak torque. ${ }^{6}$ However, in our cases, the three ankles had a good range of dorsiflexion.

After the primary midfoot amputation, the feet seemed to invert on plantar flexion due to the overactive tibialis anterior. They also developed ulceration on the 
anterolateral aspect of the closure site. Since the ankles had good range of motion without any tendoachilles tightening, the senior surgeon (PM) transposed the tibialis anterior to the cuboid bone. Following the procedure, the supination disappeared while retaining the range of motion at the ankle joint. The ulcers healed in the follow-up period of around 4 to 6 months. A tendoachilles lengthening would have increased the range of dorsiflexion, hence increasing the risk of heel ulcerations.

\section{CONCLUSION}

A tibialis anterior transposition could prove to be more effective than a tendoachilles lengthening in cases where the foot has a good range of dorsiflexion but at the same time has an inversion deformity.

\section{REFERENCES}

1. Singh N, Armstrong DG, Lipsky BA. Preventing foot ulcers in patients with diabetes. JAMA 2005 Jan 12;293(2):217-228.

2. Alexiadou K, Doupis J. Management of diabetic foot ulcers. Diabetes Ther 2012 Dec;3(1):1-15.
3. Mueller MJ, Diamond JE, Delitto A, Sinacore DR. Insensitivity, limited joint mobility, and plantar ulcers in patients with diabetes mellitus. Phys Ther 1989 Jun;69(6):453-462.

4. Lin SS, Lee TH, Wapner KL. Plantar forefoot ulceration with equinus deformity of the ankle in diabetic patients: the effect of tendo-Achilles lengthening and total contact casting. Orthopedics 1996 May;19(5):465-475.

5. Armstrong DG, Stacpoole-Shea S, Nguyen H, Harkless LB. Lengthening of the Achilles tendon in diabetic patients who are at high risk for ulceration of the foot. J Bone Joint Surg Am 1999 Apr;81(4):535-538.

6. Hastings MK, Mueller MJ, Sinacore DR, Salsich GB, Engsberg JR, Johnson JE. Effects of a tendo-Achilles lengthening procedure on muscle function and gait characteristics in a patient with diabetes mellitus. J Orthop Sports Phys Ther 2000 Feb;30(2):85-90.

7. Berry DC, Sabacinski KA, Habershaw GH, Giurini JM, Chrzan JS. Tendo Achilles procedures for chronic ulcerations in diabetic patients with transmetatarsal amputations. J Am Podiatr Med Assoc 1993 Feb;83(2):96-100.

8. Turan 1. Tarsometatarsal amputation and tibialis anterior tendon transposition to cuneiform 1. J Foot Surg 1985 MarApr;24(2):113-115. 